DOI 10.46916/02022022-1-978-5-00174-464-1

\title{
СРАВНЕНИЕ МЕТОДОВ РАСЧЕТА МИНИМАЛЬНОЙ ТОЛЩИНЫ СТЕНКИ ДЛЯ БАРАБАННОЙ ТЕХНОЛОГИИ
}

\author{
Дмитриев Константин Сергеевич \\ ведущий специалист НТЦ \\ АО «ВНИИнефть»
}

Аннотация: Барабанный метод считается одним из самых эффективных способов строительства морских трубопроводов диаметром до 457,2 мм. В связи с возникающей в перспективе потребностью в освоении Арктического шельфа необходимо искать пути оптимизации и усовершенствования «традиционных» технологий, применяемых на морских месторождениях. Применимость барабанного метода на российском арктическом шельфе может стать одним из способов сокращения капитальных вложений и минимизации работ, проводимых в море. В статье приведен сравнительный анализ расчетов минимальной толщины стенки для барабанной технологии на основании российских и зарубежных стандартов для оценки текущего состояния российской нормативной базы с целью выявления аспектов и возможных ограничений с ее стороны.

Ключевые слова: Барабанный метод, морские трубопроводы, Арктический шельф, подводные трубопроводные системы.

\section{COMPARISON OF COMPUTATION APPROACHES OF MINIMUM REELABLE WALL THICKNESS}

\section{Dmitriev Konstantin Sergeevich}

\begin{abstract}
The reel-lay method is considered one of the most effective ways to install offshore pipelines with a diameter of up to $457.2 \mathrm{~mm}$. Due to the arising need for the development of the Arctic shelf in the long run, it is necessary to look for ways to optimize and improve the "traditional" technologies used in offshore fields. The reel-lay method applicability on the Russian Arctic shelf may become one of the ways to reduce capital expenditure and minimize work carried out at sea. The article presents approaches comparative study of the minimum wall thickness
\end{abstract}


for the reel-lay method based on Russian and foreign regulations to assess the current condition of the Russian regulatory environment in order to identify aspects and feasible constraints.

Key words: Reel-lay method, offshore pipelines, Arctic shelf, submarine pipeline systems.

\section{Введение}

Мировая добыча нефти и газа все больше набирает тенденцию в направлении разработки морских месторождений. В частности, для России наиболее перспективной зоной является Арктика - нефтегазодобывающий регион, разработка которого требует высоких капиталовложений, новых технологических решений и связана с высокими рисками в условиях сурового климата.

Барабанная технология в перспективе способна снизить стоимость разработки шельфовых проектов за счет снижения трудозатрат в море и высокой скорости укладочных работ.

В связи с этим, важное значение приобретают технико-экономические показатели данного способа строительства. Одной из составляющих в капитальных затратах при строительстве трубопровода является стоимость трубной продукции. Для корректирования стоимостных показателей необходимо определить оптимальные параметры трубопровода, в частности, толщину стенки.

Основным критерием, влияющим на проектирование допустимой толщины стенки наматываемого трубопровода, является предотвращение изгиба при намотке на барабан, в результате которого может произойти смятие трубопровода. Также немаловажную роль играет накопление деформаций в результате значительных знакопеременных деформаций, начиная от намотки на береговой трубосварочной базе и заканчивая деформациями во время укладки.

Основными российскими нормативными документами, в соответствии с которыми осуществляется расчет толщины стенки морского трубопровода, являются:

- Подводные трубопроводные системы [1];

- Правила классификации и постройки морских подводных трубопроводов [2]; 
- Морские трубопроводы. Правила проектирования и строительства [3].

Данные нормативные документы, базой для которых послужил норвежский стандарт DNV-OS-F101-2000 "Submarine pipeline systems" [6], предъявляют требования к технологии на всех этапах ее реализации, начиная от процесса намотки и заканчивая укладкой на месте проведения работ.

\section{1. Расчеты}

\section{1 Стандарт «Подводные трубопроводные системы» \\ Единственным государственным документом, полностью} охватывающим барабанную технологию, является стандарт «Подводные трубопроводные системы», расчеты в котором учитывают внутреннее и внешнее давление, неблагоприятное сочетание нагрузок (природных, функциональных, случайных) и предполагают незначительные изменения свойств стали, содержат рекомендации по выбору толщины стенки трубопровода для барабанной технологии на основании комбинации внешнего избыточного давления и изгибающего момента [12, с. 4] и теории чистого изгибающего момента, но не учитывают положительный эффект, оказываемый приложенным тяговым усилием во время намотки (рис. 1) $[13$, c. 55], направленным на повышение ее эффективности и уменьшение вероятности местного изгиба, т.е. потери устойчивости.

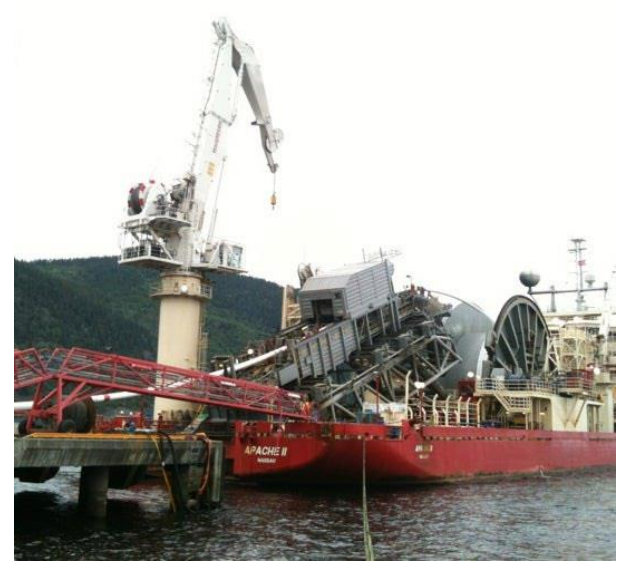

\section{Рис. 1. Процесс намотки трубопровода на трубоукладочное судно}

Кроме того, отдельные случаи присутствия концентраций деформаций, обусловленные наличием геометрических и материальных неоднородностей, например, несоответствия толщин стенок и пределов текучести соседних 
труб, следует рассматривать с использованием комбинации методов, основанных на надежности и исследовании методом конечных элементов $[9$, c. 2$]$.

Уравнения, представленные в нормативном документе «Подводные трубопроводные системы», можно преобразовать в зависимость минимальной толщины стенки трубопровода от деформаций, возникающих от изгиба, наружного диаметра и материала трубопровода.

Общий вид расчетного значения деформаций сжатия может быть выражен:

$$
\varepsilon_{d}=\varepsilon_{F} \cdot \gamma_{F} \cdot \gamma_{C}+\varepsilon_{E} \cdot \gamma_{E}+\varepsilon_{A} \cdot \gamma_{A} \cdot \gamma_{C}
$$

где: $\varepsilon_{d}-$ расчетное значение деформации сжатия, \%;

- $\varepsilon_{F}-$ деформация от функциональных нагрузок, равная номинальной изгибной деформации, \%;

- $\varepsilon_{E}-$ деформация, вызванная природными нагрузками, \%;

- $\varepsilon_{A}-$ деформация от функциональных нагрузок, \%;

- $\gamma_{F}-$ коэффициент надежности для функциональной нагрузки;

- $\gamma_{C}-$ коэффициент условий работы;

- $\gamma_{E}-$ коэффициент надежности для природной нагрузки;

- $\gamma_{A}-$ коэффициент надежности для аварийной нагрузки.

В результате намотки трубопровод подвергается деформациям сжатия, а в последствии и внутреннему избыточному давлению. Для предотвращения потери устойчивости трубопровод во всех сечениях должен удовлетворять следующему условию:

$$
\varepsilon_{d} \leq \frac{\varepsilon_{c}}{\gamma_{\varepsilon}}
$$

где: $\gamma_{\varepsilon}-$ коэффициент сопротивления деформациям;

- $\varepsilon_{c}-$ сопротивление изгибной деформации, \%, рассчитываемое по формуле:

$$
\varepsilon_{c}=0,78 \cdot\left(\frac{t}{D}-0,01\right) \cdot\left(1+5 \cdot \frac{\sigma_{h}}{f_{y}}\right) \cdot \alpha_{h}^{-1,5} \cdot \alpha_{g w},
$$

где: $t$ - номинальная толщина стенки, мм;

- $\quad D$ - номинальный наружный диаметр, мм;

- $\sigma_{h}$ - кольцевое напряжение, МПа;

- $f_{y}$ - предел текучести, МПа; 
- $\alpha_{h}$ - максимальный допускаемый коэффициент деформационного упрочнения при растяжении;

- $\alpha_{g w}-$ коэффициент кольцевого сварного шва.

- Итоговое уравнение получено при следующих принятых условиях:

- при намотке, осуществляемой на береговой трубосварочной базе, отсутствуют природные и случайные нагрузки, которые могут оказать влияние на процесс намотки;

- местное расчетное давление и наружное давление равны атмосферному, т.е. перепад давления по сечению равен нулю.

Итоговая формула после преобразования выглядит следующим образом:

$$
t \geq\left(0,01+\frac{1}{0,78} \cdot \frac{\gamma_{F} \cdot \gamma_{c} \cdot \gamma_{\varepsilon}}{\alpha_{h}^{-1,5} \cdot \alpha_{g w}} \cdot \varepsilon_{F}\right) \cdot D,
$$

Как упоминалось ранее, в основе российских нормативных документов по морским трубопроводам лежит норвежский стандарт 2000 года издания. Если рассмотреть актуальную версию DNVGL-ST-F101-2017 "Submarine pipeline systems" [5], номинальная толщина стенки определяется по формуле (с учетом условий, принятых ранее):

$$
t \geq\left(0,01+\frac{1}{0,85^{1,5}} \cdot \frac{\gamma_{F} \cdot \gamma_{c} \cdot \gamma_{\varepsilon}}{\alpha_{h}^{-1,5} \cdot \alpha_{g w} \cdot \alpha_{m a t}} \cdot \varepsilon_{F}\right) \cdot D,
$$

где: $\alpha_{\text {mat }}$ - коэффициент, применяемый для материалов, имеющих фронт Людерса.

Данные формулы справедливы только при соблюдении строгих требований, предъявляемых к материалу трубы.

\section{2 Подход Компании Technip}

Компания Теchnip пошла по другому пути и разработала собственные методы оценки толщины стенки наматываемого трубопровода, основываясь на подходе Murphey и Langner, суть которого заключалась в учете неоднородности в результате изменяющихся характеристик материала стали (предела текучести) и различий в толщине стенки с помощью коэффициента 0,5 в формуле расчета сопротивления изгибной деформации [7, с. 201]:

$$
\varepsilon_{c}=0,5 \cdot \frac{t}{D-t}
$$

Эти уравнения, основанные на теории чистого изгибающего момента [10, с. 6], достаточно просты в своем представление, учитывают геометрические параметры трубопровода, а также диаметр шейки барабана. 
Минимальная допустимая толщина стенки трубопровода для намотки при соблюдении жестких требований к техническим характеристикам труб рассчитывается по формуле:

$$
\begin{gathered}
t=\frac{2 \cdot D}{1-t_{f a b}} \cdot \varepsilon_{b-n o m}, \\
\varepsilon_{b-n o m}=\frac{D}{D_{\text {ш }}+D+2 \cdot t_{\text {из }}},
\end{gathered}
$$

где: $t_{f a b}$ - заводской допуск на толщину стенки трубы, \%;

- $t_{\text {из }}$ - толщина изоляции, мм;

- $D_{\text {ш }}$ - диаметр шейки барабана, мм;

- $\varepsilon_{b-n o m}-$ номинальная изгибная деформация, \%.

\section{2. Результаты расчетов}

\section{1 Исходные данные}

В качестве исходных данных было принято следующее:

- диапазон сравниваемых диаметров от 355,6 до 508 мм (верхний предел на текущий момент ограничен барабанной технологией и российскими нормативными документами [3, с. 28]);

- толщина изоляции для всех вариантов $-3,5$ мм;

- рассматриваемые группы прочности стали: К46, К52, К54;

- расчеты проводились для судов Seven Vega (рис. 2), Lewek Constellation, North Ocean 105 (рис. 3) и Deep Blue, которые являются передовыми в данной технологии.

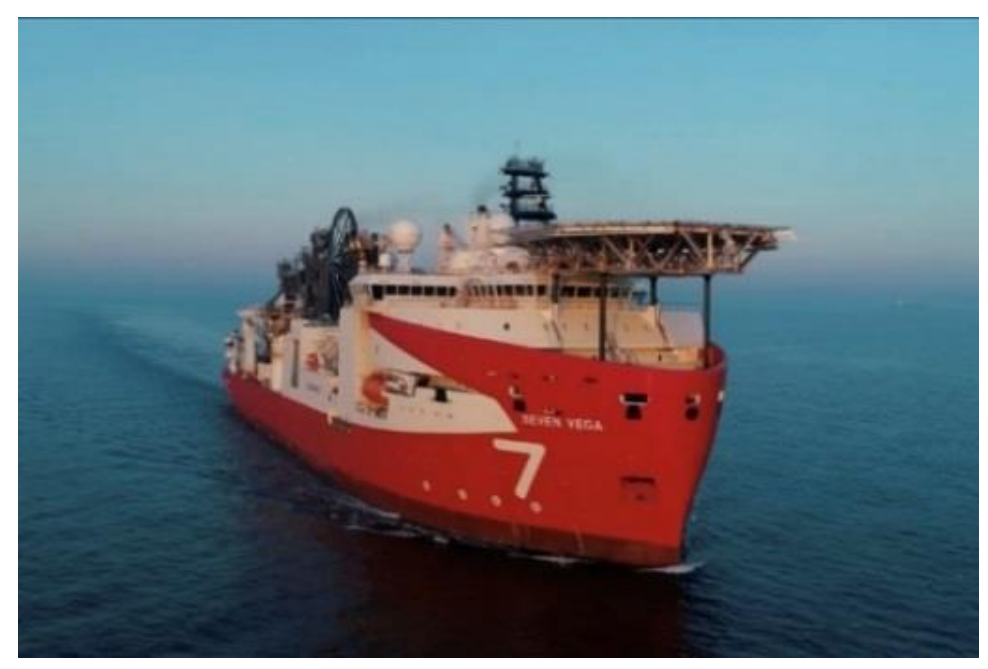

Pис. 2. Seven Vega 


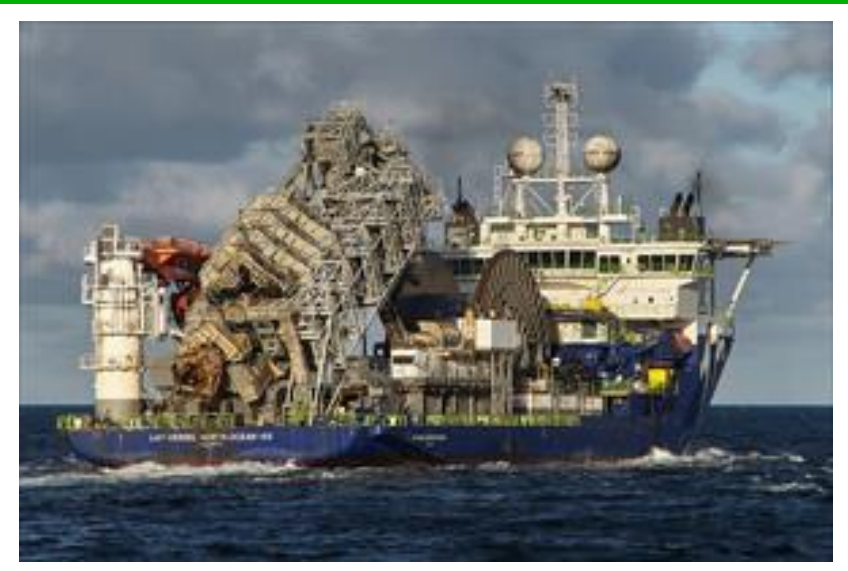

\section{Puc. 3. North Ocean 105}

\section{2 Сравнение результатов расчетов}

Сопоставительные результаты расчетов номинальных толщин стенок в соответствии с государственным стандартом «Подводные трубопроводные системы» и формулами Компании Теchnip представлены на рисунках 4-7.

На всех представленных зависимостях толщины стенок, рассчитанные в соответствии $\mathrm{c}$ российскими нормативными документами, превышают результаты Компании Тесhnip на величину от 17 до $25 \%$. Если сравнить расчеты, выполненные по актуальному норвежскому стандарту, с государственным стандартом, можно отметить небольшое различие, в среднем составляющее 0,1 мм, направленное в сторону уменьшения номинальной толщины стенки.

\section{Вывод}

Рассмотрение итоговых значений показало заметное различие в результатах расчетов по методике в соответствии с российскими нормами и подходом Компании Теchnip. Российские стандарты имеют более строгие требования, но не учитывают влияние натяжения при намотке, также согласно результатам, полученным сотрудниками Компании Technip, на данный расчет может повлиять расположение оборудования, что потребует введение поправочных коэффициентов [9, с. 6].

Превышение обусловлено наличием коэффициентов надежности и коэффициентов, учитывающих деформационное упрочнение стали в результате цикличных деформаций, что приводит к значительному запасу несущей способности трубопровода. Но при полученных данных возникает следующий вопрос, при необоснованном увеличении толщины стенки, как поведет себя материал трубопровода при знакопеременных деформациях. 
В большинстве актуальных государственных стандартах отсутствует минимальная информация о барабанной технологии. Помимо этого, стандарт, наиболее полно описывающий данный метод строительства, основан на документе, потерявшем свою актуальность десять лет назад, из чего следует необходимость актуализации и развития российской нормативной базы.

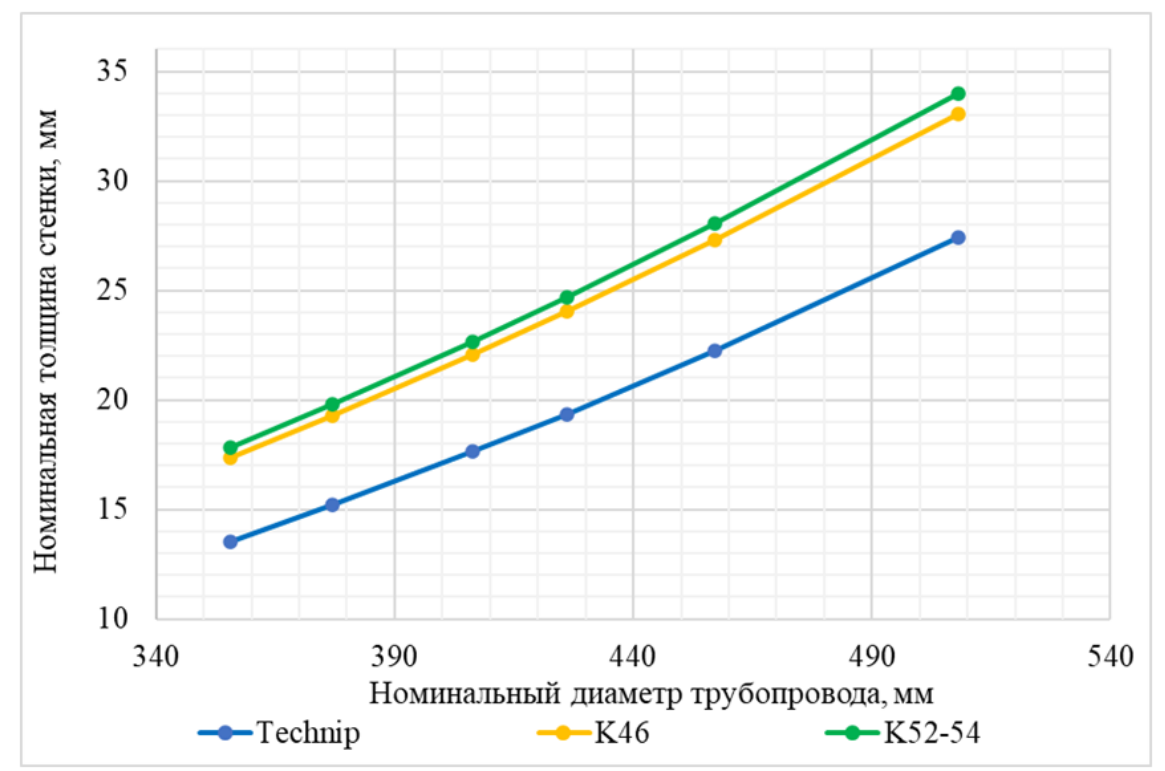

Рис. 4. Номинальная толщина стенки трубопровода для судна Seven Vega

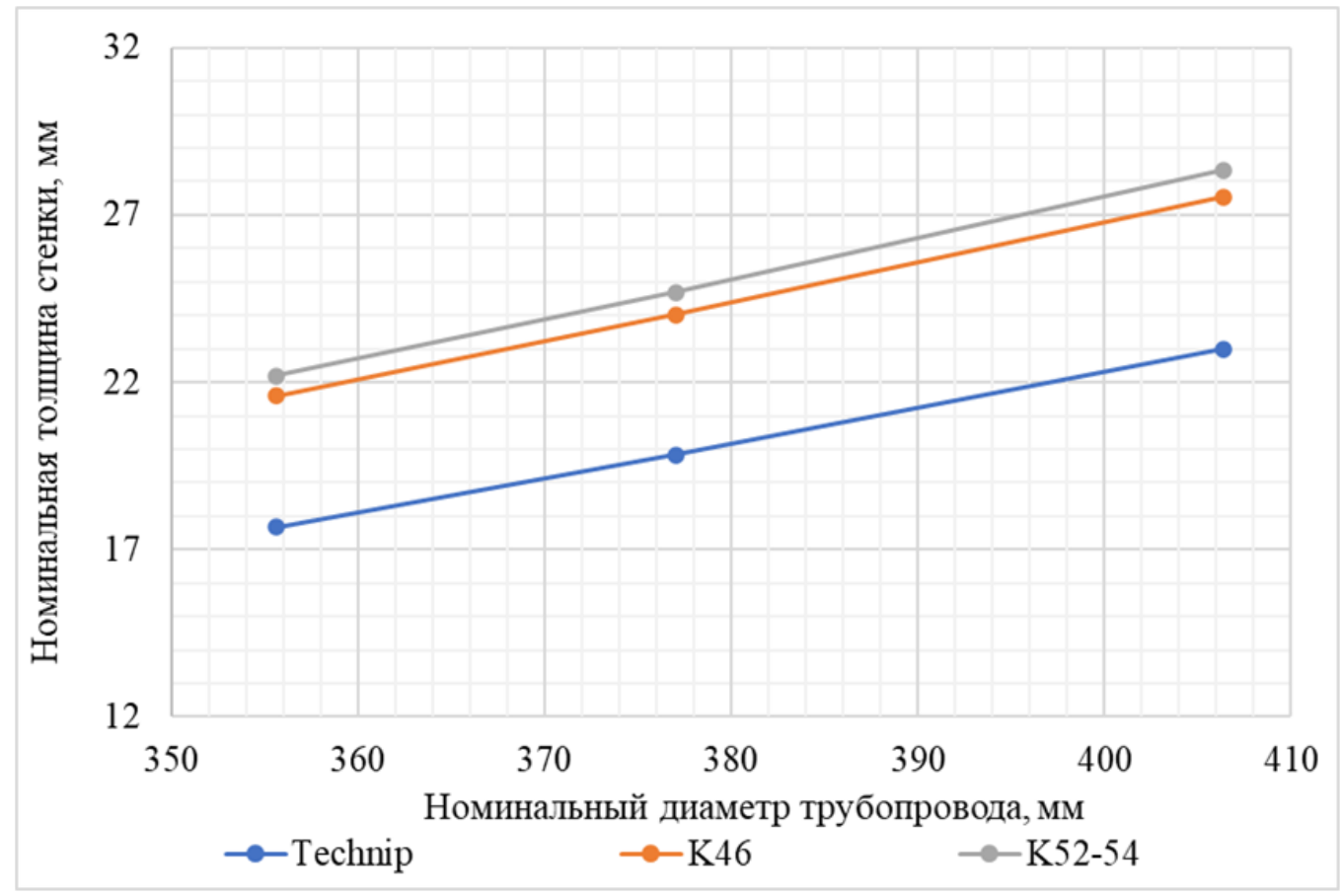

Рис. 5. Номинальная толщина стенки трубопровода для судна Lewek Constellation 


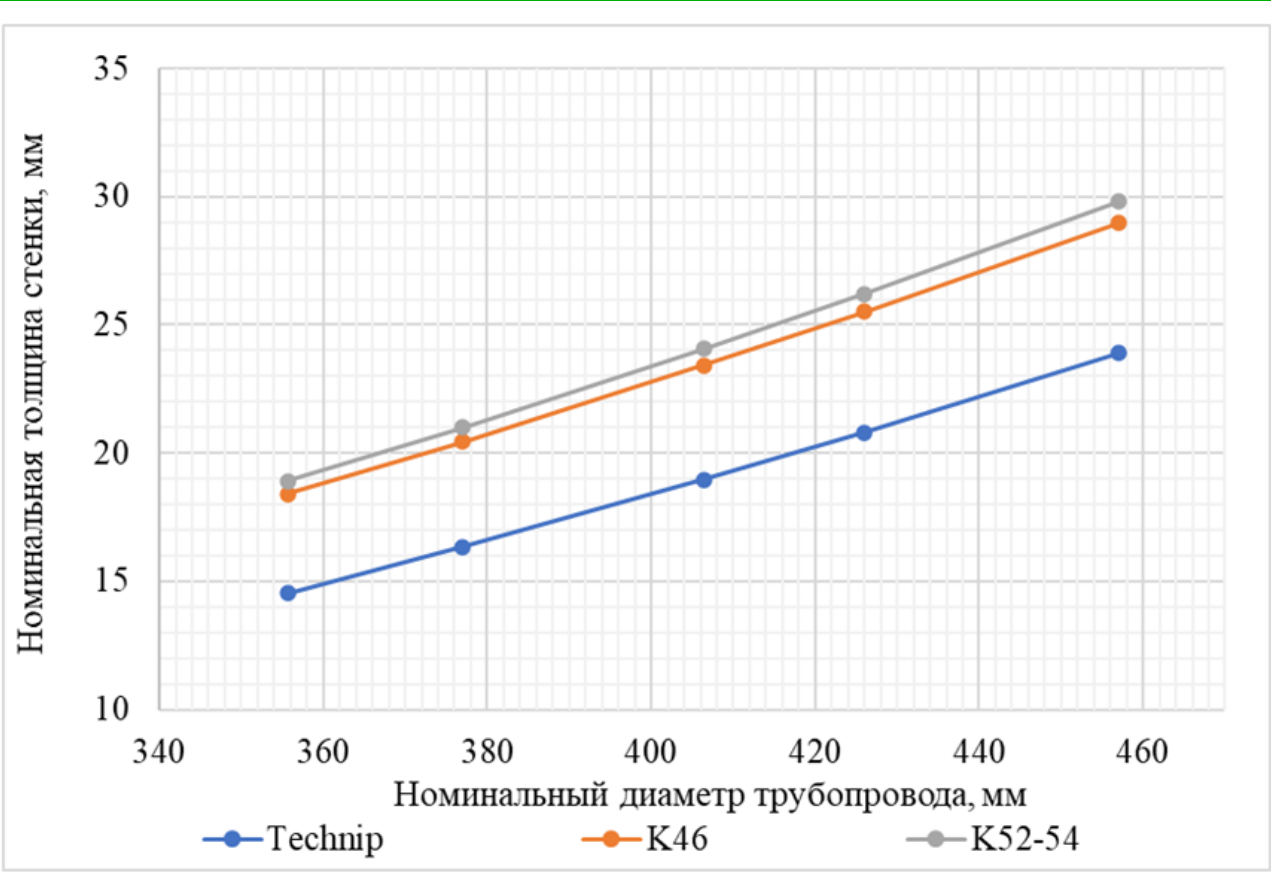

Рис. 6. Номинальная толщина стенки трубопровода для судна Deep Blue

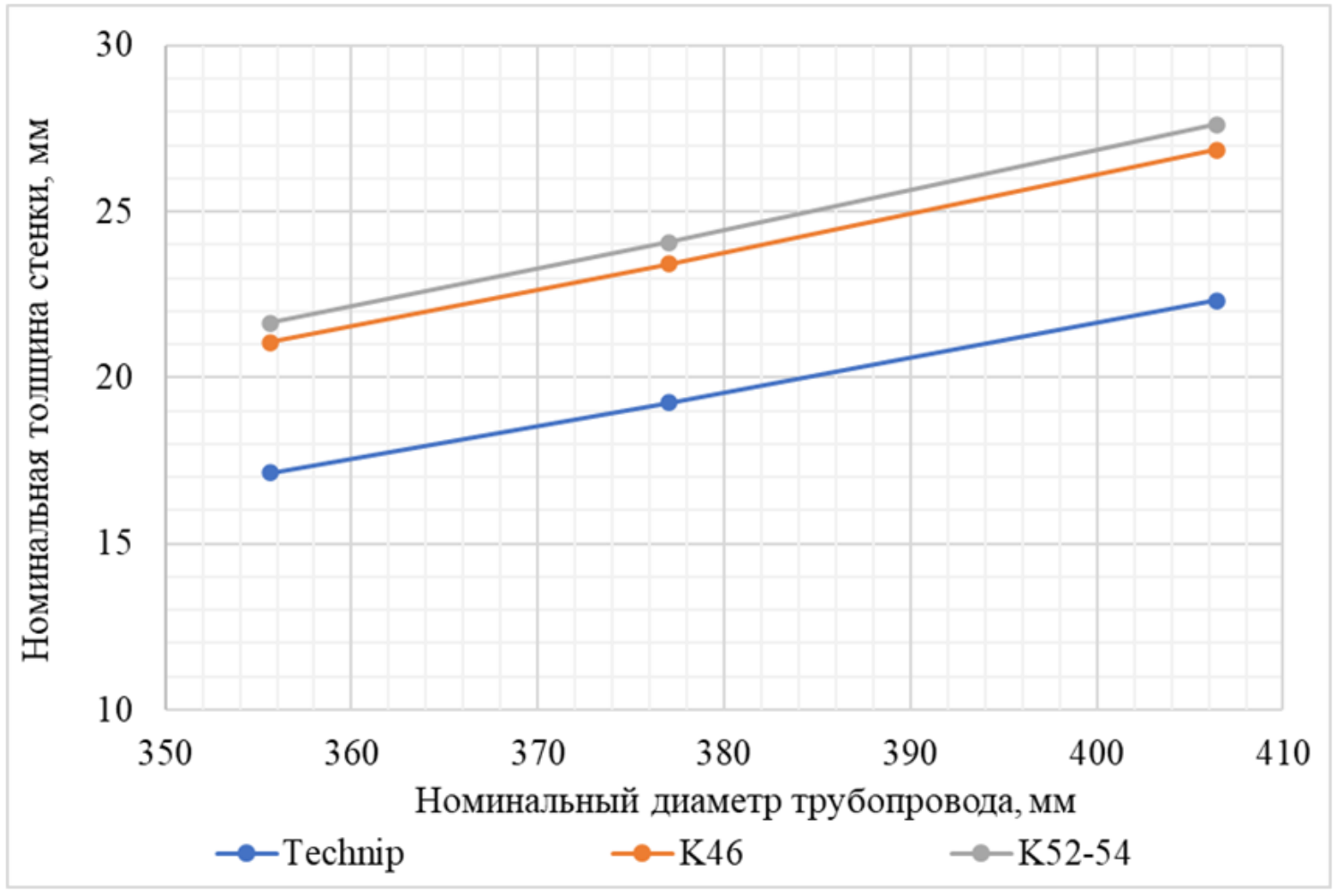

Рис. 7. Номинальная толщина стенки трубопровода для судна North Ocean 105 


\section{Список литературы}

1. ГОСТ Р 54382-2011 Подводные трубопроводные системы [Электронный ресурс]. - Режим доступа: - http://docs.cntd.ru/document/gost-r54382-2011, свободный. - Загл. с экрана.

2. НД №2-020301-004 Правила классификации и постройки морских подводных трубопровод [Электронный ресурс]. - Режим доступа: https://files.stroyinf.ru/Data2/1/4293756/4293756490.pdf, свободный. - Загл. с экрана.

3. СП 378.1325800.2017 Морские трубопроводы. Правила проектирования и строительства [Электронный ресурс]. - Режим доступа: https://docs.cntd.ru/document/550965722, свободный. - Загл. с экрана.

4. СТО Газпром 2-3.7-050-2006 Подводные трубопроводные системы [Электронный ресурс]. - Режим доступа: - https://elima.ru/docs/?id=7877, свободный. - Загл. с экрана.

5. DNVGL-ST-F101 Submarine pipeline systems [Электронный ресурс]. Режим доступа: - https://www.academia.edu/Submarine_pipeline_systems, свободный. - Загл. с экрана.

6. DNV-OS-F101 Submarine pipeline systems [Электронный ресурс]. Режим доступа: - http://www.opimsoft.com/download/reference/os-f101_200510.pdf, свободный. - Загл. с экрана.

7. Chen J.F., Ooi J.Y., Teng J.G. (Editors) Structures and Granular Solids: From Scientific Principles to Engineering Application / J.F. Chen, J.Y. Ooi, J.G. Teng. - London.: CRC Press, 2008. - 340 p.

8. Denniel S. Optimising Reeled Pipe Design through Improved Knowledge of Reeling Mechanics / S. Denniel // Offshore Technology Conference, 2009. $13 \mathrm{p}$.

9. Denniel S., Tkaczyk T., Howard B., Levold E., Aamlid O. The Development of a Refined Assessment Procedure for the Pipeline Reeling Process Using Reliability and Finite Element Techniques / S. Denniel, T. Tkaczyk, B. Howard, E. Levold, O. Aamlid // International Conference on Ocean, Offshore and Arctic Engineering, 2009. - 9 p.

10. Denniel S., Tkaczyk T., Smith D. Reelable Cost Effective and Enabling Pipeline Technologies / S. Denniel, T. Tkaczyk, D. Smith // Offshore Technology Conference, 2011. - $16 \mathrm{p}$. 
11. Karjadi E., Smienk H., Boyd H., Aamlid O. Extend Reel-ability of HMC New Aegir Reeling Vessel Based on Reliability Based Assessment and Bending Tests Program / E. Karjadi, H. Smienk, H. Boyd, O. Aamlid // International Conference on Ocean, Offshore and Arctic Engineering, 20012. - 16 p.

12. Manouchehri S. A Discussion of Practical Aspects of Reeled Flowline Installation / S. Manouchehri // International Conference on Ocean, Offshore and Arctic Engineering, 2012. - 12 p.

13. Manouchehri S., Howard B., Denniel S. A Discussion of the Effect of the Reeled Installation Process on Pipeline Limit States / S. Manouchehri, B. Howard, S. Denniel // International Offshore and Polar Engineering Conference, 2008. - 7 p. 